\title{
Pixel's History based Background Subtraction Parameters Tuning using Fuzzy Inference System
}

\author{
Lajari Alandkar \\ Ph.D. Scholar, \\ Department of \\ Electronics Engineering, \\ Walchand Institute of Technology, \\ $\mathrm{MH}$, India
}

\author{
Sachin R. Gengaje \\ HOD, \\ Department of \\ Electronics Engineering, \\ Walchand Institute of Technology, \\ $\mathrm{MH}$, India
}

\begin{abstract}
Object detection is one of the challenging steps in video surveillance. The most popular and robust technique for object detection is background subtraction. It is always challenging to obtain better performance of background subtraction algorithm as it requires appropriate initial tuning of common parameters like number of components in Gaussian Mixture Model (GMM), threshold, learning rate and initial values. Traditional way of tuning is manual selection of parameters based on background scenario. It requires good understanding of background scene to the end user and iterative experimentation with manual setting leading to significantly time intensive and tedious tuning process. As initial tuning affects performance of background subtraction, it makes significant impact on usage of an algorithm and its selection based on current application. In this paper, simplified novel methodology of pixel's history based parameter tuning is proposed. Method uses statistical features to approximate background situation and fuzzy logic approach to bound tuning criteria. Broadly, statistical features are extracted from pixel's history and processed by Fuzzy Inference System (FIS). GMM parameters as FIS output are exclusively used for background subtraction. Algorithm evidently demonstrates its effectiveness in parameter selection. The proficiency of proposed tuning system is also highlighted by comparison with manual and Particle Swarm Optimization (PSO) based method over diverse Wallflower Dataset.
\end{abstract}

\section{General Terms}

Pattern Recognition, Computer Vision

\section{Keywords}

Object Detection, Background Subtraction, Gaussian Mixture Model, Fuzzy Inference System.

\section{INTRODUCTION}

Object detection in video streams is preliminary step of information extraction in many computer vision applications, including video surveillance, people tracking, traffic monitoring, law enforcement and military services. Performance of object detection phase has strong influence on higher level operation like classification, tracking, activity recognition etc. [1]. There are few conventional methods of object detection like background subtraction, frame differencing, optical flow, feature point statistic and classification, feature point matching and tracking [2]. Amongst all techniques, background subtraction is widely popular because of less complexity and computational time [3] but main problem is its sensitivity to dynamic changes in background which causes consequent need of background adaptation and maintenance [4]. Over the years research has progressed towards improving robustness and accuracy against dynamic changes incurred due to complex background conditions like sudden or slow illumination change, snow, rain, waving tree, flickering monitors, bootstrap, camouflage etc. $[3,4]$.

A common attributes of background subtraction algorithms are learning rates, thresholds, and initial values that must be tuned in order to produce desired accuracy [6]. Tuning process for preferred parameters are less attentive because of lack of awareness and time intense repeated experimentation with manual method to achieve optimum result. It is challenging as well because it requires understanding of background situation, parameters need to be managed globally instead at a pixel level, necessity of expert knowledge and different setting for several scenario. All these aspects cumulatively limits use and selection of background subtraction methods based on application [7].

Lot of literature have addressed various background techniques like Min-Max inter-frame difference, single Gaussian model, Gaussian mixture model (GMM), Kernel density estimation, Eigen background and code book [5]. Amongst all, GMM based background subtraction is widely popular because of its multimodality, stability, robustness and ability to handle different critical situations and provide reliable result $[3,6,7,8]$. Major research has been carried out on intrinsic improvement into GMM model related to number of component(K), learning rate $(\alpha)$, Threshold(T), initialization of GMM parameters (i.e. weight, mean and variance) [3].

The selection of pixel wise $\mathrm{K}$ is suggested by Zivkovic [9] and Cheng et al. [10]. Zivkovic [9] proposes an online algorithm that selects number of Gaussians using Dirichlet prior for each pixel. In same idea, Cheng et al. [10] propose a stochastic approximation procedure which is used to obtain asymptotically optimal number of Gaussians. Setting and adaptation of pixel wise $\mathrm{K}$ is presented by Shimada et al. [11], Tan et al. [12], Carminati et al. [13]. All of these works improve computation time of background subtraction because of optimal pixel wise selection of $\mathrm{K}$ but it has less effect on accuracy.

White et al. [7] proposed to automatically tune learning rate $\alpha$ and background threshold parameter T using Particle Swarm Optimization. Bowden et al. $[14,15,16]$ proposed to use a different learning rate between steps of parameters initialization and parameters maintenance. Haque et al. [17, $18,19]$ replace ' $\mathrm{T}$ ' with another less sensitive parameter (i.e. threshold ' $\mathrm{S}$ ') for foreground background separation. Result shows better performance than the original MOG.

Initialization and maintenance of other GMM parameters i.e. weight, mean and variance are discussed by Lee [20], Pavlidis 
et al. [21], Zhang et al. [22], Amintoosi et al. [23].

Analyzing above research implies that only PSO method considered initial tuning of significant parameter; whereas rest of the research mainly concerned with selection and adaptation of single parameters. It means that, parameter tuning still requires more attention in order to improve accuracy of object detection. PSO method tunes learning rate and threshold. It requires careful creation of ground truth image, repetitive expensive fitness function call which is time intensive and few assumptions about search space for tuning. Therefore, PSO method is difficult to use in real time although it improves accuracy of object detection system. Therefore, it is valuable to develop a technique which can be implemented real time, tunes important parameters, and be able to produce parameters that works well over variety of scenes. The automated technique needs to be computationally fast and without human intervention (as ground truth image creation in PSO) so as to implement in real time. In this paper, considering all these factors as an objective, distinctive algorithm based on pixel's history using Fuzzy Inference System (FIS) is proposed. It tunes significant parameters like learning rate, threshold, and number of Gaussian component. Parameters' setting by applying tuning criteria based on background situation is primarily used to develop this technique. Statistical features are extracted from pixel's history for easy and fast background understanding. Further step to get GMM parameters from statistical features requires nonlinear mapping. FIS is considered for solving nonlinear mapping as against Artificial Neural Network (ANN). It gives significant advantage in computational time space over ANN. Moreover linguistic, vague and imprecise tuning criteria can be efficiently handled by fuzzy logic approach. As well as, FIS has closeness to human reasoning; so solutions obtained is easy to apply and understand. FIS does not require formal model of problem and training data for solving problems [24]. These special attributes of FIS supports its selection against ANN. FIS outputs are tuned GMM parameters which can be used to train GMM and obtain satisfactory result. Proposed system is validated by experimentation and comparison with manual and PSO tuning method.

This paper is structured in five sections. Second section describes GMM parameters and their tuning criteria. Section three demonstrates proposed work with detail subsections include extraction of statistical features based on pixel's history and design of fuzzy inference system to process statistical features. Section four presents experimentation, results and discussion. Paper concluded in fifth section.

\section{GMM PARAMETERS}

There are many parameters of GMM based background subtraction algorithm such as learning rate, number of Gaussians, threshold (minimum background ratio), initial Gaussian weight, initial Gaussian standard deviation and standard deviation threshold [7, 8]. It is found that selection of Number of Gaussians (K), Threshold (T) and Learning rate $(\alpha)$ plays important role in performance of background subtraction algorithm. As $\mathrm{K}$ and $\mathrm{T}$ account for multimodality; ' $\alpha$ ' for adaptation to background change. Details of their selection criteria are discussed further.

Number of Gaussian K defines complexity of background in terms of multimodality. It is represented by integer value ranging from 2 to 5 . For simple indoor scenes, a small value of $\mathrm{K}$ is sufficient, perhaps $\mathrm{K}=2$; A larger $\mathrm{K}$ (i.e., 3, 4, 5) is needed for outdoor complex scenes like snow, rain, waving tree, wind etc. $[6,7]$.
Threshold $\mathrm{T}$ is measure of maximum consideration of multimodal behavior in background scene. It is fraction between background distribution and foreground distribution. Hence, it is selected on the basis of dynamic changes in scene. Small value of $\mathrm{T}$ is selected for uni-modal background model i.e. simple background with less dynamic changes. If background has multi-modal distribution then $\mathrm{T}$ must be selected higher. $[6,7]$

The learning rate $\alpha$ defines speed of adaptation of background. Reciprocal of learning rate gives the number of frames after which GMM parameters are updated. It is most significant parameter which affects overall performance of background subtraction; as it controls agility of adaptation of algorithm. In a simple scene, small value of $\alpha$ is sufficient because of less dynamic changes. Complex outdoor scene requires adequate value of $\alpha$ which must be high enough to accommodate multimodality of background scene, but low enough to detect slowly moving foreground objects $[6,7]$. Selection of learning rate is challenging when dealing with such complex scenes [7].

\section{PROPOSED WORK}

Proposed GMM parameter tuning algorithm uses pixel's history based on initial video sequence (150 to 200 frames are sufficient) to derive statistical features. These features are then processed using FIS. Tuned GMM parameters from FIS are further used to train GMM and achieve acceptable result over further video. General block diagram of proposed work is shown in Fig.1



Fig 1: The schematic of proposed work

Following subsections briefly explains design of statistical parameters based on pixel's history and design of fuzzy inference system for background understanding and tuning respectively.

\subsection{Pixel's History Based Features}

Pixel's history is developed by initial video sequence contains purely background (i.e. without moving foreground object). Four Statistical features are derived from pixel history. Feature 1 and 2 perceive multimodality (because of snow, rain, waving tree, flickering monitor etc.), Feature 3 accommodate random behavior (simultaneous effect of shadow, illumination change and other uncertain changes), and overall illumination change over the history of background are interpreted with Feature 4.

Proposed work considers Grey scale video for implementation. Initially, deviation history for each pixel in frame is obtained using initial video sequence.

Let us consider ' $\mathbf{P}$ ' as one of the pixels in background represented by vector of pixel intensities in its temporal history. ' $\mathbf{p}$ ' is subset of ' $\mathrm{P}$ ' representing vector of selective 
pixels intensities depending on frame rate ' $r$ ' of video. Thus, use of ' $p$ ' for statistical feature calculation reduces overload of deviation calculation (e.g. if frame rate is $10 \mathrm{fps}$ then every $10^{\text {th }}$ pixel from $\mathbf{P}$ will be considered in $\mathbf{p}$ ).

$P=\left\{P_{0}, P_{1}, P_{2}, \ldots \ldots, P_{N-1}\right\}$

$p=\left\{p_{0}, p_{1}, p_{2}, \ldots, p_{N / r}\right\}$

$$
\text { Where, } p_{i}=P_{i \times r} \quad 0 \leq i \leq N / r
$$

$\mathrm{N}$ : Total number of initial frame used to build pixel's history. It should be a number between 150 to 200 and multiple of $\mathbf{r}$.

$r$ : Frame rate of video

Deviation history (differences in successive pixels value in temporal history) of pixel is denoted by $\mathbf{D}$ and it is obtained by vector $\mathbf{p}$

$D=\left\{d_{0}, d_{1}, d_{2}, \ldots, d_{\frac{N}{r}-1}\right\}$

Where $d_{j}=p_{j+1}-p_{j}$

$$
0 \leq j \leq\left(\frac{N}{r}-1\right)
$$

Four statistical features are derived from above history. All features are scalar.

Multimodal behavior in scene is caused because of snow, rain, waving tree etc. It means pixels facing such situations under constant illumination condition can show two intensity values alternately in temporal history. It divides deviation history into nearly equal number (fifty-fifty) of positive and negative deviation. Therefore, Feature 1 represent amount of background with approximate 50\% (45-55) positive deviation and Feature 2 is amount of background with approximate 50\% (45-55) negative deviation

- Feature 1: Percentage of total pixels in background with deviation history of $45-55 \%$ positive deviation. It is obtained by two steps

a. Use deviation history in equation (3) to calculate percentage of positive deviation for single pixel in background. Denoted by PPD (percentage positive deviation)

PPD for single pixel $=$

$\frac{\text { No of positive Deviation in deviation history }}{\frac{N}{r}-1} \times 100$

b. Feature 1 defined as

Feature $1=$

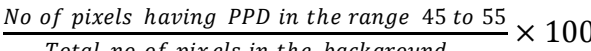

- Feature 2: Percentage of total pixels in background with deviation history of 45-55\% negative deviation. The steps are same as above to calculate Feature 2

a. Use deviation history in equation (3) to calculate percentage of negative deviation for single pixel in background. It is denoted by PND (percentage negative deviation)

PND for single pixel $=$

$\frac{\text { No of negative Deviations in deviation history }}{\frac{N}{r}-1} \times 100$

b. Feature 2 is given by
Feature $2=$

$\frac{\text { No of pixels having PND in the range } 45 \text { to } 55}{\text { Total no of pixels in the background }} \times 100$

Feature 3 defines situation of constant brightness. It indicates percentage of pixels in background facing constant illumination most (at least 70\%) of the time. High value of Feature 3 specifies that most part of the background is constant while low value shows it is multimodal or random or both.

- Feature 3: Percentage of total pixels in background with deviation history of at least $70 \%$ zero deviation. The steps are quite same again to obtain Feature 3.

a. Use deviation history in equation (3) to calculate percentage of zero deviation for single pixel in background. Represented by PZD (percentage zero deviation)

PZD for single pixel $=$

$\frac{\text { Number of zero Deviations in deviation history }}{\frac{N}{r}-1} \times 100$

b. Feature 3 can be obtained by

Feature $3=$

$\frac{\text { No of pixels having PZD above } 70}{\text { Total no of pixels in the background }} \times 100$

Feature 4 extract amount of illumination change all over the background in fixed time in terms of overall standard deviation. Slow illumination change causes low overall standard deviation. It leads to low value of Feature 4. High illumination change cause increase in overall standard deviation. It will lead to high value of Feature 4

- $\quad$ Feature 4: Average of pixel's standard deviation. It is obtained by two steps

a. Standard deviation of each pixel is calculated over its temporal history based on equation (1). Indicated by PSD (pixel standard deviation)

Standard Deviation of the Pixel $(P S D)=$ $\sqrt{\frac{1}{N} \sum_{i=0}^{N-1} P_{i}-\bar{P}}$

Where, $\bar{P}$ : temporal mean of pixel and given by,

b. Feature 4 indicates,

$$
\bar{P}=\frac{1}{N} \sum_{i=0}^{N-1} P_{i}
$$

Feature $4=\frac{1}{L} \sum_{i=0}^{L-1} P S D_{i}$

Where, L: Total number of pixels in background

Feature 1, 2 and 3 together has been used to tune $\mathrm{K}$ and $\mathrm{T}$ whereas Feature 4 is used to decide learning rate ' $\alpha$ '.

\subsection{Fuzzy Inference System (FIS) Design}

FIS is used to implement GMM parameters tuning criteria. The schematic diagram of Fuzzy Inference System (FIS) is shown in Fig. 2. FIS uses statistical features as crisp input in order to tune GMM parameters as crisp output. A typical FIS performs mapping using four basic components: fuzzifier, rule base, inference engine and defuzzifier. Set of statistical feature is represented by $F$ which is used for tuning GMM parameter set represented by $\mathrm{G}$. Here $\mathrm{F}=\{\mathrm{F} 1, \mathrm{~F} 2, \mathrm{~F} 3, \mathrm{~F} 4\} \mathrm{T}$ and $\mathrm{G}=\{\mathrm{K}, \mathrm{T}$, alpha $\} \mathrm{T}$. 


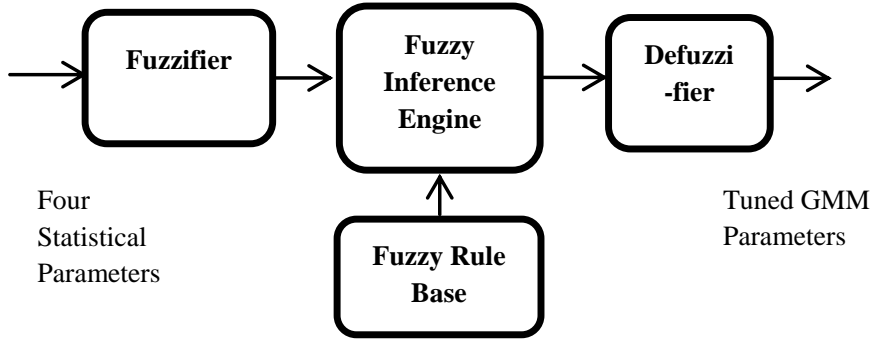

Fig 2: The schematic of Fuzzy Inference System

Input statistical features and output GMM parameters are treated as fuzzy variables. Gaussian membership functions (MFs) are used for specifying fuzzy sets to define input and output variables because of smoothness and concise notation. Gaussian function is described by two parameters mean and standard deviation. Mean of fuzzy set varies for each membership function of every input and output variable. Uncertainty associated with each membership function of variable is represented by standard deviation. Proposed FIS is briefly explained according to basic blocks.

\section{Fuzzification}

The procedure for fuzzification is as follows,

- The fuzzy sets corresponding to each input variable $F_{1}$, $\mathrm{F}_{2}, \mathrm{~F}_{3}, \mathrm{~F}_{4}$ are generated by taking mean and standard deviation of membership functions. Proposed system considers five membership functions for each input/output variable named as very low, low, medium, high, very high.

- For every statistical parameter $F_{i}$, degree of membership in the fuzzy set is calculated. Thus, input fuzzy set is obtained for all input variable

\section{Fuzzy Rule base}

Fuzzy rule base consists of 35 simple rules described using fuzzy rule table. It is compact way of representing rules in the form of table. Fuzzy rules for proposed FIS and their rule tables are explained in two steps as follows.

\section{- $\quad$ Fuzzy rules for GMM parameters K, T:}

Number of Gaussians (K) and Threshold (T) depends on $F_{1}$, $F_{2}$ and $F_{3}$. It is important to note that $K$ and $T$ must vary simultaneously. Because, $\mathrm{K}$ is number of Gaussian distribution and $\mathrm{T}$ is measure of minimum number of distribution considered for background. It means, if $\mathrm{K}$ is selected high to account multimodality then $\mathrm{T}$ needs to be set high for inclusion of more distribution in background model.

$\mathrm{F}_{1}$ and $\mathrm{F}_{2}$ together extract multimodality. Their values are high for complex scenario. Whereas, according to tuning criteria $\mathrm{K}$ and $\mathrm{T}$ must be high for complex background. Therefore, rule can be developed as e.g.

\section{IF $F_{1}$ is high AND $F_{2}$ is high THEN $K$ is high and $T$ is high.}

Table 1 represents fuzzy rule table for $K$ and $T$ w.r.t $F_{1}$ and $F_{2}$. Five rules along the main diagonal of table strongly support multimodality in background, so they carry high weightage. While, rest of the rules carry low weight as they are less concerned with multimodality.

Along with multimodality, static or random behaviour of background is considered for selection of $\mathrm{K}$ and $\mathrm{T}$. This behaviour is extracted by $F_{3}$. If it is high then most background is static. If it is low, then most background may be multimodal or random or both in nature. However, according to tuning criteria $\mathrm{K}$ and $\mathrm{T}$ must be set to low for static scene and high for multimodal or random scene. Therefore, rule can be written as an e.g.

\section{IF F3 is low THEN $\mathrm{K}$ is high and $\mathrm{T}$ is high}

Five simple rules are developed based on this concept. Table 2 shows the fuzzy rule table for K, T w.r.t Feature 3. All rules are equally weighted.

Table 1. Fuzzy Rule Table for $K$ and $T$ based on $F_{1}, F_{2}$. (Responses of input and output variable are VL-Very Low, L-Low, M-Medium, H-High, VH - Very High)

\begin{tabular}{|c|c|c|c|c|c|c|}
\hline & \multicolumn{5}{|c|}{ Feature 1} \\
\hline & & VL & $\mathbf{L}$ & $\mathbf{M}$ & $\mathbf{H}$ & $\mathrm{VH}$ \\
\hline \multirow{5}{*}{ 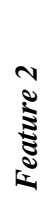 } & VL & VL & $\mathrm{L}$ & $\mathrm{M}$ & $\mathrm{H}$ & $\mathrm{VH}$ \\
\hline & L & $\mathrm{L}$ & $\mathrm{L}$ & $\mathrm{M}$ & $\mathrm{H}$ & $\mathrm{VH}$ \\
\hline & $\mathbf{M}$ & $\mathrm{M}$ & $\mathrm{M}$ & $\mathrm{M}$ & $\mathrm{H}$ & $\mathrm{VH}$ \\
\hline & $\mathbf{H}$ & $\mathrm{H}$ & $\mathrm{H}$ & $\mathrm{H}$ & $\mathrm{H}$ & $\mathrm{VH}$ \\
\hline & VH & $\mathrm{VH}$ & $\mathrm{VH}$ & $\mathrm{VH}$ & $\mathrm{VH}$ & $\mathrm{VH}$ \\
\hline
\end{tabular}

Table 2. Fuzzy Rule Table for K, T based on Feature 3

\begin{tabular}{|l|l|l|l|l|l|}
\hline Feature 3 & VL & L & M & H & VH \\
\hline K, $\boldsymbol{T}$ & VH & H & M & L & VL \\
\hline
\end{tabular}

- $\quad$ Fuzzy rules for GMM parameter $\alpha$ :

Learning rate is selected based on rate of illumination change in background. This information is extracted by $\mathrm{F}_{4}$ i.e. if a background illumination change fast then $F_{4}$ is high. Therefore, learning rate is set relatively to Feature 4. Rule can be defined as an e.g.

\section{IF $F_{4}$ is high THEN learning rate is high.}

Five simple rules are developed based on this concept. Table 3 shows fuzzy rule table for learning rate $\alpha$. All rules are equally weighted.

\section{Table 3. Fuzzy Rule Table for $\alpha$ based on Feature 4}

\begin{tabular}{|l|l|l|l|l|l|}
\hline Feature 4 & VL & L & M & H & VH \\
\hline$\alpha$ & VL & L & M & H & VH \\
\hline
\end{tabular}

\section{Fuzzy Inference Engine:}

The inference engine defines mapping from input fuzzy sets into output fuzzy sets. It is possible that one or more rules may fire at same time

- It determines the degree to which antecedent is satisfied for each rule.

- If antecedent of a given rule has more than one clause, fuzzy operators are applied to obtain one number that represents result of antecedent for that rule. AND fuzzy operator is used for proposed FIS which select minimum of two membership value.

- Implication method is applied for shaping of consequent (output fuzzy set), based on antecedent. MINIMUM implication method is used for proposed system which truncates output fuzzy set of each rule.

Output fuzzy set of all rules are aggregated by commonly used MAXIMUM method. Three aggregated output fuzzy sets are obtained corresponds to $\mathrm{K}, \mathrm{T}$ and alpha. 


\section{Defuzzification}

It maps aggregated output fuzzy sets into a crisp number. Several methods for defuzzification are in practice, including centroid, maximum, mean of maxima, height, and modified height defuzzifier. The most popular defuzzification method is centroid, which calculates and returns center of gravity of aggregated fuzzy set [27]. Therefore, centroid is used as defuzzifier in proposed FIS. Three crisp numbers are obtained corresponds to $\mathrm{K}, \mathrm{T}$ and $\alpha$ by defuzzification processes which can be used for training GMM.

Threshold (T), Learning rate $(\alpha)$ are directly referred from FIS output; whereas, $\mathrm{K}$ must be rounded to nearest integer before training GMM.

\section{EXPERIMENTAL RESULT AND DISCUSSION}

Object detection system using adaptive GMM is built with reference to the paper [8].This system takes pre-processed (filtered to remove Gaussian noise) video as an input and produces a foreground mask sequence as output. It is further post processed to remove speckle noise by morphological closing operation. Experimentation is done over canonical sequences in the Wallflower Dataset. Following subsections present brief description about Wallflower dataset, performance result over experimentation and discussion about proposed work.

\subsection{Dataset}

Wallflower database is open source database [25]. It includes seven set of image sequences covering possible critical situations in background. All sequences are of size 160x120 pixels, sampled at $4 \mathrm{~Hz}[25,26]$. Brief descriptions of sequences are as below:

- Moved Object (MO) - A person enters into a room, makes a phone call, and leaves. The phone and the chair are left in a different position.

- Time of Day (TOD) - The light in a room gradually changes from dark to bright. Then, a person enters the room and sits down.

- $\quad$ Light Switch (LS) - A room scene begins with the lights on. Then a person enters the room and turns off the lights for a long period. Later, a person walks in the room, switches on the light, and moves the chair, while the door is closed.

- Waving Trees (WT) - A tree is swaying and a person walks in front of the tree.

- Camouflage (C) - A person walks in front of a monitor, which has rolling interference bars on the screen. The bars include similar colour to the person's clothing.

- Bootstrapping (B) - The image sequence shows a busy cafeteria and each frame contains people.

- Foreground Aperture (FA) - A person with uniformly colour shirt wakes up and begins to move slowly.

\subsection{Experimental Result}

In this experimentation, GMM based object detection system is tuned using proposed method and its performance is evaluated. Brief description of experimentation steps is as below

1. GMM parameters (i.e. K, T, $\alpha$ ) are obtained by proposed method using initial video sequence covering corresponding background situation in the Wallflower dataset. Other background subtraction parameters such as number of training frame, initial Gaussian standard deviation are set to appropriate value.

2. Object detection system is tuned using resultant parameters values and executed.

3. Output of the system is binary foreground mask which is evaluated against ground truth foreground mask provided in the Wallflower dataset.

Performance evaluation is made in terms of total error and overall accuracy (Average of accuracy over all sequences). It is important to note that bootstrap (B) image sequences is excluded while experimentation because initial sequence without foreground object could not be available.

Experimentation result for each type of sequence is shown in Table 5 and it is compared against other tuning methods such as Manual, PSO1 and PSO2. Visual result for each type of sequence by all tuning methods is shown in Fig.3. Short descriptions for other tuning methods are as below

- Manual Method [26]: In this case, same parameter set for different sequences is used for tuning GMM which gives optimal result over dataset after repetitive manual adjustment.

- PSO1[7] : Optimized parameter set is used to tune GMM, which is achieved by maximizing fitness function through recursive call.

- $\quad$ PSO2 [7]: It is same as PSO1, the difference is only that GMM is tuned with different setting of parameters for each sequence.

\subsection{Discussion}

GMM with proposed tuning method has total error rate 10700 and overall accuracy of $90.71 \%$. It can be observed from Table 5 and Fig. 3 that performance of GMM with proposed tuning method reduces total error and improves the overall accuracy as compared to manual setting or PSO1; whereas results are equivalent with PSO2 optimization method. Recommended method generates different GMM parameters setting w.r.t background scene based on its history. It avoids constraint of initial assumption and use of ground truth image. It reduces overload of repetitive manual setting or recursive function call for optimal tuning. All these features prove effectiveness of proposed tuning method in real time use against PSO2. This method also automates tuning because of these special attributes. Recommended method does not intend to claim that it provides optimal parameter set however it performs comparable to other optimization techniques with several additional advantages. Table 4 summarizes all features of proposed method.

Table 4. Attribute based comparison of PSO2 and proposed tuning method

\begin{tabular}{|l|l|l|}
\hline Attributes & PSO2 & Proposed \\
\hline $\begin{array}{l}\text { Background } \\
\text { subtraction } \\
\text { parameter can be } \\
\text { tuned }\end{array}$ & $\mathrm{T}, \alpha$ & $\mathrm{K}, \mathrm{T}, \alpha$ \\
\hline Parameter Sets & $\begin{array}{l}\text { Different for each } \\
\text { Sequence }\end{array}$ & $\begin{array}{l}\text { Different for } \\
\text { each Sequence }\end{array}$ \\
\hline $\begin{array}{l}\text { Ground Truth image } \\
\text { required while } \\
\text { tuning }\end{array}$ & Necessary & Not required \\
\hline
\end{tabular}




\begin{tabular}{|l|l|l|}
\hline $\begin{array}{l}\text { Initial Assumption } \\
\text { required before } \\
\text { tuning }\end{array}$ & Necessary Not required \\
\hline $\begin{array}{l}\text { Repetitive Call to } \\
\text { certain function for } \\
\text { appropriate tuning }\end{array}$ & Necessary & Not required \\
\hline $\begin{array}{l}\text { Way to use of } \\
\text { tuning method for } \\
\text { GMM }\end{array}$ & $\begin{array}{l}\text { Real time use } \\
\text { possible after } \\
\text { offline tuning }\end{array}$ & $\begin{array}{l}\text { Real time as well } \\
\text { as offline tuning } \\
\text { can be possible }\end{array}$ \\
\hline Scope & $\begin{array}{l}\text { Initial global tuning } \\
\text { image } \\
\text { available/created for truth } \\
\text { video sequence }\end{array}$ & $\begin{array}{l}\text { Initial global } \\
\text { tuning for any } \\
\text { video sequence } \\
\text { except bootstrap }\end{array}$ \\
\hline
\end{tabular}

\section{CONCLUSION}

The performance of GMM using recommended tuning method is evaluated over six different scenarios. Total error rate is 10700 and overall accuracy is $90.71 \%$. Comparative study proves that, proposed method significantly reduces total error rate as compared to manual tuning method and performs equally to optimization based tuning method (PSO) with additional advantages.

In this paper, novel method of pixel's history based GMM parameter tuning is established. It automatically tunes significant GMM parameters (i.e. $\mathrm{K}, \mathrm{T}, \alpha$ ) for various scenes and provides satisfactory result. It approximates background condition with four statistical features and generates GMM parameters set according to tuning criteria using fuzzy approach. Simple approach avoids necessity of repetitive experimentation, initial assumption, and ground truth image for tuning. These special attributes prove proficiency of proposed technique in real time use. Easy parameter tuning eliminates the obstacle over the use of background subtraction algorithm and its selection based on application. This method provides basic framework for background understanding of various scenarios and tuning of background subtraction parameters.

Current research gives instincts for few more future works. It may include tuning and adaptation of GMM parameter based on scene history, pixel level tuning and adaption of GMM parameter using pixel level statistical features, inclusion of additional rules or statistical features in basic framework for identification and tuning of bootstrap situation..

\section{REFERENCES}

[1] Jun-Wei Hsieh, Shih-Hao Yu, Yung-Sheng Chen, An Automatic Traffic Surveillance System for Vehicle Tracking and Classification, IEEE Transactions on Intelligent Transportation Systems, Vol. 7,

[2] S. Kannan, A. Sivasankar, Moving Object Detection Based on Fuzzy Color Histogram Features and Dynamic Threshold Optimization, The International Journal of Science \& Technoledge, Vol 2 Issue 3 March, 2014

[3] Thierry Bouwmans, Fida El Baf, Bertrand Vachon. "Background Modeling using Mixture of Gaussians for Foreground Detection - A Survey". Recent Patents on Computer Science, Bentham Science Publishers, 2008, 1 (3), pp.219-237.

[4] Lucia Maddalena, Alfredo Petrosino, "A fuzzy spatial coherence-based approach to background/ foreground separation for moving object detection", Neural Comput \& Applic, 2010
[5] Yannick Benezeth, Pierre-Marc Jodoin, Bruno Emile, Helene Laurent, Christophe Rosenberger, "Comparative study of background subtraction algorithms". Journal of Electronic Imaging, Society of Photo-optical Instrumentation Engineers, 2010

[6] Qi Zang and Reinhard Klette, "Parameter Analysis for Mixture of Gaussians Model", www.citr.auckland.ac.nz/researchreports/CITR-TR188.pdf

[7] Brandyn White B, Shah M. Automatically tuning background subtraction parameters using particle swarm optimization. IEEE Int Conf on Multimedia \& Expo (ICME 2007), Beijing, China, 2007; 1826-1829.

[8] Stauffer C, Grimson W. "Adaptive background mixture models for real-time tracking". Proc. IEEE Conf. on Comp Vision and Patt. Recog.(CVPR 1999) 1999; 246252

[9] Zivkovic Z. "Improved adaptive Gaussian mixture model for background subtraction". Int Conf Pattern Recognition (ICPR 2004), 2004, 2: 28-31.

[10] Cheng J, Yang J, Zhou Y, Cui Y. Flexible background mixture models for foreground segmentation. J Image Vision Comput (IVC 2006) 2006; 24: 473-482.

[11] Shimada A, Arita D, Taniguchi R. Dynamic control of adaptive mixture-of-gaussians background model. AVSS 2006, Sydney, Australia, November 2006, 5.

[12] Tan R, Huo H, Qian J, Fang T. Traffic video segmentation using adaptive-k gaussian mixture model. The Int Workshop on Intelligent Computing (IWICPAS 2006), Xi'An, China, August 2006, 125-134.

[13] Carminati L, Benois-Pinau J. Gaussian mixture classification for moving object detection in video surveillance environment. IEEE Int Conf on Image Processing (ICIP 2005), September 2005, 113- 116.

[14] KaewTraKulPong P, Bowden R. An improved adaptive background mixture model for real-time tracking with shadow detection. Proceedings 2nd European Workshop on Advanced Video Based Surveillance Systems (AVBS 2001), Kingston, UK, September 2001.

[15] KaewTraKulPong P, Bowden R. Adaptive visual system for tracking low resolution color targets. BMVC 2001 , Manchester UK, September 2001, 1: 243-252.

[16] KaewTraKulPong P, Bowden R. A real-time adaptive visual surveillance system for tracking low resolution color targets in dynamically changing scenes. J Image Vision Comput 2003; 21(10): 913-929

[17] Haque M, Murshed M, Paul M. On stable dynamic background generation technique using Gaussian Mixture models for robust object detection. 5th IEEE Int Conf On Advanced Video and Signal Based Surveillance, AVSS 2008, 2008

[18] Haque M, Murshed M, Paul M. A hybrid object detection technique from dynamic background using gaussian mixture models. IEEE Int Workshop on Multimedia Signal Processing, MMSP 2008, Cairns, Queensland, Australia, October 2008.

[19] Haque M, Murshed M, Paul M. Improved Gaussian Mixtures for robust object detection by adaptive multibackground generation. Int Con on Pattern Recognition, 
ICPR 2008, Tampa, Florida, USA, December 2008.

[20] Lee D. Online adaptive Gaussian mixture learning for video applications. Workshop on Statistical Methods for Video Processing (SMVP 2004), Prague, Czech, May 2004; 105-116.

[21] Morellas V, Pavlidis I, Tsiamyrtzis P. DETER: Detection of events for threat evaluation and recognition. Mach Vision Appl 2003; 15: 29-45.

[22] Zhang Y, Liang Z, Hou Z, Wang H, Tan M. An adaptive mixture gaussian background model with online background reconstruction and adjustable foreground mergence time for motion segmentation. ICIT 2005, December 2005; 23-27.

[23] Amintoosi M, Farbiz F, Fathy M, Analoui M, Mozayani N. QR decomposition-based algorithm for background subtraction. ICASSP 2007, April 2007, 1: 1093-1096.
[24] Swati Chaudhari, Manoj Patil, "Study and Review of Fuzzy Inference Systems for Decision Making and Control", American International Journal of Research in Science, Technology, Engineering \& Mathematic, issue 5, Feb 2014, pp. 88-92

[25] Wallflower

Dataset: http://research.microsoft.com/users/jckrumm/WallFlower /TestImages.htm

[26] Toyama K, Krumm J, Brumitt B, Meyers B. Wallflower: Principles and practice of background maintenance. Int. Conf. on Computer Vision, (ICCV 1999), Corfu, Greece, September 1999; 255 -261

[27] Fundamentals of Logic Concepts (2011). Retrieved from http://ptgmedia.pearsoncmg.com/images/0135705991/sa mplechapter/0135705991.pdf

\section{APPENDIX}

Table 5. Result of six canonical sequences (MO, TD, LS, WT, C and FA) over GMM for manual, PSO1, PSO2 and proposed tuning method

\begin{tabular}{|l|c|c|c|c|c|c|c|c|c|}
\hline $\begin{array}{l}\text { Algorithm w/Tuning } \\
\text { Method }\end{array}$ & Metric & MO & TD & LS & WT & C & FA & $\begin{array}{c}\text { Total } \\
\text { Error }\end{array}$ & $\begin{array}{c}\text { Overall } \\
\text { Accuracy }\end{array}$ \\
\hline GMM w/Manual & FN & 0 & 1008 & 1633 & 1323 & 398 & 2442 & & \\
Method[26] & FP & 0 & 20 & 14169 & 341 & 3098 & 530 & $\mathbf{2 4 9 6 2}$ & $\mathbf{7 8 . 3 3}$ \\
\hline GMM w/PSO 1 [7] & FN & 0 & 807 & 1716 & 43 & 2386 & 2392 & & \\
& FP & 0 & 6 & 772 & 1689 & 1463 & 572 & $\mathbf{1 1 8 4 6}$ & $\mathbf{8 9 . 7 1}$ \\
\hline GMM w/PSO 2 [7] & FN & 0 & 737 & 2059 & 219 & 929 & 2300 & & \\
& FP & 0 & 9 & 96 & 267 & 433 & 631 & $\mathbf{7 6 8 0}$ & $\mathbf{9 3 . 3 3}$ \\
\hline GMM w/Proposed & FN & 20 & 456 & 85 & 536 & 436 & 634 & & $\mathbf{1 0 7 0 0}$ \\
Method & FP & 0 & 153 & 2457 & 852 & 2646 & 2425 & $\mathbf{9 0 . 7 1}$ \\
\hline
\end{tabular}

\begin{tabular}{|c|c|c|c|c|c|c|}
\hline & MO & TD & LS & WT & $\mathbf{C}$ & FA \\
\hline Test & & & & & & \\
\hline Ground Truth & & & & & & \\
\hline $\begin{array}{l}\text { GMM } \\
\text { w/Manual method }\end{array}$ & & & & & & \\
\hline GMM w/PSO1 & & & & & & \\
\hline GMM w/PSO2 & & & & & & \\
\hline $\begin{array}{l}\text { GMM } \\
\text { w/Proposed Method }\end{array}$ & & & I & & & \\
\hline
\end{tabular}

Fig.3 Experimental Result of GMM with different tuning method over Wallflower Dataset 